\title{
Modulation of Gene Expression and Cytoskeletal Dynamics by the Amyloid Precursor Protein Intracellular Domain (AICD) $D$
}

\author{
Thorsten Müller, ${ }^{*+}$ Caoimhin G. Concannon, ${ }^{\ddagger}$ Manus W. Ward, ${ }^{\ddagger}$ Ciara M. Walsh, $\neq$ \\ Anca L. Tirniceriu, ${ }^{* \S}$ Florian Tribl, ${ }^{+}$Donat Kögel,, Jochen H.M. Prehn, ${ }^{\ddagger I}$ \\ and Rupert Egensperger*II
}

\begin{abstract}
*Institute of Pathology and Neuropathology, University Hospital Essen, D-45122 Essen, Germany;
‡Department of Physiology and Medical Physics and RCSI Neuroscience Research Centre, Royal College of Surgeons in Ireland, Dublin 2, Ireland; "Experimental Neurosurgery, Center for Neurology and Neurosurgery, Johann Wolfgang Goethe University Clinics Frankfurt, D-60590 Frankfurt, Germany; ${ }^{+}$Medical Proteome-Center, Ruhr-University Bochum, D-44780 Bochum, Germany; and SInstitute of Neuropathology, University Hospital Muenster, D-48149 Muenster, Germany
\end{abstract}

Submitted April 7, 2006; Revised August 14, 2006; Accepted October 26, 2006

Monitoring Editor: Paul Forscher

\begin{abstract}
Amyloidogenic processing of the amyloid precursor protein (APP) results in the generation of $\beta$-amyloid, the main constituent of Alzheimer plaques, and the APP intracellular domain (AICD). Recently, it has been demonstrated that AICD has transactivation potential; however, the targets of AICD-dependent gene regulation and hence the physiological role of AICD remain largely unknown. We analyzed transcriptome changes during AICD-dependent gene regulation by using a human neural cell culture system inducible for expression of AICD, its coactivator FE65, or the combination of both. Induction of AICD was associated with increased expression of genes with known function in the organization and dynamics of the actin cytoskeleton, including $\alpha 2$-Actin and Transgelin (SM22). AICD target genes were also found to be differentially regulated in the frontal cortex of Alzheimer's disease patients compared with controls as well as in AICD/FE65 transiently transfected murine cortical neurons. Confocal image analysis of neural cells and cortical neurons expressing both AICD and FE65 confirmed pronounced changes in the organization of the actin cytoskeleton, including the destabilization of actin fibers and clumping of actin at the sites of cellular outgrowth. Our data point to a role of AICD in developmental and injury-related cytoskeletal dynamics in the nervous system.
\end{abstract}

\section{INTRODUCTION}

The amyloid precursor protein (APP) is a type- 1 transmembrane protein composed of a large extracellular and a small intracellular domain and has been widely implicated in the pathogenesis of Alzheimer's disease (AD). APP can undergo proteolytic cleavage by $\beta$ - and $\gamma$-secretase activities, resulting in the production of $\beta$-amyloid ( $A \beta$ ) (Selkoe, 1994). $\mathrm{A} \beta$ is the main constituent of amyloid plaques and is thought to be neurotoxic by inducing oxidative stress, inflammation, and neurodegeneration (Mattson, 2004). The intraneuronal accumulation of $\mathrm{A} \beta$ protofibrils is thought to cause progressive neurotoxicity in cortical neurons (Hartley et al., 1999). In addition, the secretion of soluble $\mathrm{A} \beta$ oligomers inhibits hippocampal long-term potentiation and alters the memory of complex learned behavior (Walsh et al., 2005).

\footnotetext{
This article was published online ahead of print in $M B C$ in Press (http://www.molbiolcell.org/cgi/doi/10.1091/mbc.E06-04-0283) on November 8, 2006.

$\square$ The online version of this article contains supplemental material at $M B C$ Online (http://www.molbiolcell.org).

II These authors contributed equally to this work.

Address correspondence to: Thorsten Müller (thorsten.t.mueller@rub.de).
}

The APP intracellular domain (AICD), a small 6-kDa protein, originates from APP cleavage mediated by $\gamma$-secretase activity (Octave et al., 2000). This cleavage can occur after Val636 (AICD59), Ala638 (AICD57), or Leu645 (AICD50) corresponding to the $\gamma$ - or $\varepsilon$-cleavage site (Figure 1A) of the $\gamma$-secretase complex (Sastre et al., 2001; Yu et al., 2001). More recently, AICD was shown to have transactivation potential (Cao and Sudhof, 2001). AICD levels are detectable in membrane fractions of murine total brain homogenates, and they increase significantly in mice overexpressing the Swedish mutation of human APP (Ryan and Pimplikar, 2005). Moreover, Hirano bodies found in the degenerating neurons of Alzheimer's patients stain positive with antisera raised against the cytoplasmic domain of APP (Munoz et al., 1993), suggesting an accumulation of AICD during Alzheimer's disease.

Processing of the $\mathrm{C}$ terminus of APP and generation of AICD has been suggested to closely resemble $\gamma$-secretasemediated cleavage of Notch, effecting the release of the Notch intracellular domain (NICD) from its membrane anchor (Artavanis-Tsakonas et al., 1999), its subsequent translocation to the nucleus, and the activation of NICD-dependent gene expression. Expression of exogenous AICD in baby hamster kidney, COS cells, or in primary neurons also demonstrates a predominant nuclear localization (Cupers et al., 2001; Gao and Pimplikar, 2001; Kimberly et al., 2001; 
A

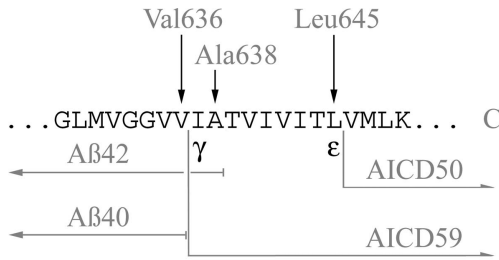

B

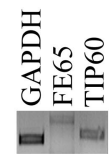

Figure 1. Generation of AICD isoforms. (A) The C terminus of APP can be cleaved by $\gamma$-secretase activity at different sites, resulting in several AICD isoforms. For this work, AICD50 corresponding to the $\varepsilon$-site and AICD59 corresponding to the $\gamma$-site were studied. Neuroblastoma SHEP-SF cells were used to establish a cell culture model inducible for AICD (AICD50 or AICD59), its binding factor FE65, or both AICD (AICD50 or AICD59) and FE65. (B) RT-PCR analysis of mRNA from SHEP-SF cells revealed endogenously expression of FE65 and TIP60, which play an important role for AICD-dependent transactivation.

Walsh et al., 2003). The function of AICD as a signaling protein has been suggested to be dependent on the interaction of AICD with several transcriptional coactivators. It has been demonstrated that the central YENPTY motif of AICD binds to the phosphotyrosine binding domain 2 (PTB2) of the adapter protein FE65 (McLoughlin and Miller, 1996), rescuing AICD from its rapid degradation mediated by the proteasome (Nunan et al., 2001, 2003) or by insulin-degrading enzyme (Edbauer et al., 2002; Farris et al., 2003). The nuclear AICD-FE65 dimer is able to bind the histone acetyltransferase tat-interactive protein (TIP60) (Cao and Sudhof, 2001) or the transcription factor CP2/LSF/LBP1 (Zambrano et al., 1998) at the PTB1 domain of FE65, which might be sufficient for a transcriptionally active complex.

Several recent studies have reported putative target genes differentially regulated by an AICD-containing complex. Candidate genes include the prostate cancer antimetastasis gene KAI1 (Baek et al., 2002), glycogen synthase kinase $3 \beta$ (GSK3 $\beta$ ) (Kim et al., 2003), $\beta$-APP cleaving enzyme (BACE) (von Rotz et al., 2004), APP (von Rotz et al., 2004), and neprilysin (MME) (Pardossi-Piquard et al., 2005). However, regulation of some of these genes is controversial (Ryan and Pimplikar, 2005; Hebert et al., 2006). The aim of our study was to use an unbiased genome-wide analysis to identify AICD-dependent target genes. For this reason, we established AICD- and AICD/FE65-inducible cell lines and analyzed their gene expression pattern after protein induction.

\section{MATERIALS AND METHODS}

\section{Plasmid Construction}

All expression vectors for the generation of stable cell lines based on a pBI-EGFP (Clontech, Mountain View, CA) plasmid with modifications in the multiple cloning site. For the p_iA50 and p_iA59 vectors, AICD isoforms (AICD50 resp. AICD59) were generated by polymerase chain reaction (PCR) amplification introducing $\mathrm{XhoI}$ and NheI restriction sites. A vector containing wtAPP was used as template (gift from C. Kaether, LMU Munich, Munich, Germany). As required, start-, stop-codon, and a Kozak sequence were introduced by the primer. PCR fragments were ligated into XhoI-NheI-digested modified pBI-EGFP vector. For $\mathrm{p}$ _iF vector, the FE65 coding sequence (digested from pFE65myc-pCIneo received from T. Russo, CEINGE, Naples, Italy) was introduced into PvuII-linearized vector pBI-EGFP. For the doubleinducible constructs $\mathrm{p}_{\mathrm{i}} \mathrm{iFA} 50$ and $\mathrm{p}$ iFA59, the appropriate AICD isoforms were cloned into EheI-linearized p_iF vector. An additional internal ribosome entry site (IRES) element was inserted between AICD and FE65 coding sequence to allow both proteins to be translated independently from the same mRNA.

AICD50-EGFP fusion vector was generated for analysis of cellular localization of AICD. AICD50 was amplified with primers introducing XhoI and EcoRI restriction site and cloned into pEGFP-N1. All constructs were verified by sequencing.

\section{Cell Culturing, Transfection, Generation of Stable Cell Lines, and Induction of Ectopic-inserted Genes}

The human neuroblastoma cell line Tet21 (derived from SHEP-SF, kind gift from M. Schwab, DKFZ Heidelberg, Heidelberg, Germany) containing the Tet transactivator (Tet-Off system) was maintained in RPMI 1640 medium containing $10 \%$ fetal bovine serum, penicillin, and streptomycin. All transfections were performed using Lipofectamine 2000 (Invitrogen, Karlsruhe, Germany). Transfections were carried out in six-well tissue culture dishes for generation of stable lines. Briefly, cells were plated at $70 \%$ confluence the day before transfection. Cells were transfected with $3 \mu \mathrm{g}$ of plasmid of interest and $1 \mu \mathrm{g}$ of pBabe-Puro for selection. Seventy-two hours posttransfection, cells were replated to $10-\mathrm{cm}$ tissue culture dish and maintained in $1 \mu \mathrm{g} / \mathrm{ml}$ puromycin for selection and $1 \mu \mathrm{g} / \mathrm{ml}$ doxycycline (dox) to repress expression of transfected genes. Stable clones were picked after 10-20 d in culture. Expression of ectopic inserted genes was induced by washing cells twice and transferring them to culture dishes with and without dox. Plated cells were washed after 3 and 24 h. After 48 h, cells were washed twice and replated in culture dishes containing corresponding medium and washed $3 \mathrm{~h}$ later. Use of this protocol resulted in high-level expression of the ectopic-inserted genes. Earlier time points revealed lower expression levels, whereas later time point showed similar induction levels. Therefore, the 72-h time point was used for all experiments. For AICD localization experiments AICD-EGFP or control vector (pEGFP-N1) were transiently transfected in SHEP-SF cells maintained in 24-well plates (1 $\mu \mathrm{g}$ /well) using Metafectene (Biontex, Munich, Germany) as per manufacturer's protocol.

\section{Localization of AICD}

Twenty-four hours after transfection, cells were fixed and stained with Hoechst 33342. Green fluorescent protein (GFP) emission pictures were collected. AICD-EGFP-transfected cells showed predominantly nuclear staining, whereas enhanced green fluorescent protein (EGFP) control vector-transfected cells were stained through the whole cell (see Supplemental Figure). Interestingly, transfection rates were low, and just low-level-expressing AICD-EGFP-transfected cells survived $24 \mathrm{~h}$. This supports the discussed toxicity of AICD (Bertrand et al., 2001; Kim et al., 2003), which might be increased as a result of higher stability when expressed as fusion protein. FE65 immunostaining in iFA clones revealed nuclear staining of FE65 (data not shown).

\section{AICD Detection}

For detection of AICD peptide, 72-h-induced iA or iFA cells were collected in AICD lysis buffer ( $1 \%$ NP-40, $150 \mathrm{mM} \mathrm{NaCl}, 2$ mM EDTA, 2 mM 1,10 phenanthroline, $250 \mu \mathrm{g} / \mathrm{ml}$ insulin, and protease inhibitor mix [Roche Diagnostics, Mannheim, Germany]). Insulin was added to overcome AICD degradation by insulin degrading enzyme as described by Edbauer et al. (2002). The lysate was incubated at $37^{\circ} \mathrm{C}$ for $1 \mathrm{~h}$, chilled on ice for $10 \mathrm{~min}$, and subsequently centrifuged. The supernatant was immunoprecipitated with C8 antibody (kind gift of D. J. Selkoe, Center for Neurologic Diseases, Harvard University, Boston, MA). Eluated AICD peptide was analyzed by Western blotting (C8 antibody) by using $17.5 \%$ Tris-Tricine gel followed by detection using ECL reagent (Amersham Biosciences, Freiburg, Germany)

\section{Oligonucleotide Microarray Analysis}

Affymetrix GeneChip analysis was performed according to the instructions of the Expression Analysis Technical Manual (Affymetrix, Santa Clara, CA). Cells (iFA50 clone 8, iA50 clone 6 , and iA50 clone 9) were grown in flasks under conditions with and without dox for $72 \mathrm{~h}$. Total RNA was extracted at $80 \%$ confluence by using the RNeasy kit (QIAGEN, Hilden, Germany). Double-stranded cDNA was synthesized from total RNA by using Superscript Choice System (Invitrogen) and a T7-(dT)24 primer [59-GGCCAGTGAATTGTAATACGACTCACTATAGGGAGGCGG-(dT)24-39]. After phenol/chloroform extraction of double-stranded cDNA, synthesis of biotin-labeled cRNA was performed using the ENZO BioArray HighYield RNA Transcript Labeling kit (ENZO Life Sciences, Farmingdale, NY). After purification with RNeasy kit (QIAGEN), ethanol precipitation, and quantifying, the biotinylated cRNA was fragmented by alkaline treatment. Then, $15 \mu \mathrm{g}$ of adjusted fragmented cRNA in a hybridization cocktail containing eukaryotic hybridization controls was hybridized to a single GeneChip Probe Array (U133A) for $16 \mathrm{~h}$. After washing and staining, the probe arrays were scanned three times in an Affymetrix GeneChip scanner.

Raw data (cel-files) were analyzed using GeneData Expressionist software (GeneData, Basel, Switzerland). First, cel-files were processed using gradient correction. Dark and bright spots were detected, and affected genes were excluded from the analysis (Expressionist Quality Module). For normaliza- 
tion, all signals were divided by the logarithmic mean of the chip signal and multiplied by a constant to put the signals back into usual range. Thus, all experiments will have a logarithmic mean corresponding to this constant. Significant expression differences were examined using statistical criteria ( $\mathrm{N}$-fold regulation factor $>1.8 ; t$ test $<0.05$ ). For a complete list of identified genes, see Supplemental Table. Supplemental data describing the results of our GeneChip studies are deposited with GEO database at http://www. ncbi.nlm.nih.gov/geo/(accession GSE4097).

\section{Real-Time Polymerase Chain Reaction (PCR)}

To quantitate potential AICD target genes as well as AICD and FE65 induction, SYBR Green assays were performed on a GeneAmp 5700 Sequence Detection System (Applied Biosystems, Foster City, CA) with the same clones that had been used for microarray analysis (iFA50 clone 8, iA50 clone 6, and iA50 clone 9) as well as additional clones (iFA50 clone 6, iFA59 clone 16, iA59 clone 100 , iF clone 5 , iF clone 15, iEGFP clone 9, and iEGFP clone 22). In total, quantitative PCR (qPCR) analysis was performed in three iFA clones (two iFA50 and one iFA59 clones), three iA clones (two iA50 and one iA59 clones), two iF clones, and two iEGFP clones. Total RNA was isolated as described above. Template cDNA was synthesized from $2 \mu \mathrm{g}$ of total RNA using the OmniScript Reverse Transcriptase kit (QIAGEN) and the T7-(dT)24 primer, following manufacturer's instructions. Sense and antisense primer sequences will be given upon request. Cycling conditions were $50^{\circ} \mathrm{C}$ for $2 \mathrm{~min}, 95^{\circ} \mathrm{C}$ for $10 \mathrm{~min}$, followed by 40 cycles of $95^{\circ} \mathrm{C}$ for $15 \mathrm{~s}$ and $60^{\circ} \mathrm{C}$ for $1 \mathrm{~min}$. Gene expression ratios for each single clone (regulation factors, SD) were calculated according the ddCt method (Livak and Schmittgen, 2001), normalized against glyceraldehyde-3-phosphate dehydrogenase. Experiments were performed on two independent dates in quadruplicate. Melting curve analysis confirmed that only one product was amplified. Specificity was confirmed by gel electrophoresis of PCR products showing only one product with each primer set. For quantification of RNA levels in murine cortical neurons, corresponding murine primers for TAGLN, TPM1, and ACTA2 were used.

\section{Analysis of Human Brain Tissue from Alzheimer Disease Patients and Controls}

All cases had been collected at the Institute of Neuropathology, University Hospital Muenster (Muenster, Germany). Before autopsies, we obtained consent from patients' families to use samples for research. The neuropathological diagnosis of $\mathrm{AD}$ was made according to established criteria (Braak and Braak, 1991; Mirra et al., 1991). The control group consisted of brains from cases without neurological and neuropathological abnormalities. RNA was harvested from frozen postmortem samples of the frontal gray matter of each nine individuals with Alzheimer's disease and age matched controls by using TRIzol reagent (Invitrogen) according to the manufacturer's protocol. Samples were homogenized using a microdismembrator. Before cDNA synthesis RNA quality was analyzed by gel electrophoresis. Only samples with acceptable RNA quality were used for cDNA synthesis (5 AD cases [mean age 75.2 years, range 63-86, mean postmortem time $13.4 \mathrm{~h}$, and range 5-24 h] and 6 age and sex-matched controls [mean age 75.5 years, range 63-92, mean postmortem time $11.5 \mathrm{~h}$, and range $5-23 \mathrm{~h}]$ ). There were no significant differences between mean ages $(p=0.96$; analysis of variance [ANOVA] $)$ and mean intervals between death and freezing of the brain $(\mathrm{p}=0.91$; ANOVA).

For immunohistochemical analysis of $\alpha 2$-Actin (ACTA2), a monoclonal mouse-anti-human smooth muscle actin antibody (clone 1A4, M0851; Dako Denmark A/S, Glostrup, Denmark) was used. Immunostaining was carried out on 3-mm-thick paraffin wax sections (tissue from nonaffected brains) with an automated staining device (Autostainer; Dako Denmark A/S). Antigen retrieval was carried out with $0.01 \mathrm{M}$ citrate buffer at $\mathrm{pH} 6.0$ for $20 \mathrm{~min}$ in a hot water bath $\left(97^{\circ} \mathrm{C}\right)$. The primary antibody was incubated for $30 \mathrm{~min}$ at a dilution of 1:100. Antibody visualization was performed with a commercially available antibody antimouse IgG detection kit (RealEnVision [K5007]; Dako Denmark A/S)

\section{Phalloidin Staining and Immunocytochemistry}

Cells were grown to $\sim 60 \%$ confluence on glass coverslips. After induction, cells were fixed with $3.7 \%$ paraformaldehyde in phosphate-buffered saline (PBS) for $10 \mathrm{~min}$ at room temperature and permeabilized with $0.1 \%$ Triton X-100 in PBS for $5 \mathrm{~min}$ at room temperature. Cells were incubated with 10 $\mu \mathrm{g} / \mathrm{ml}$ phalloidin-tetramethylrhodamine B isothiocyanate (TRITC) (Chemie, Deisenhofen, Germany) in PBS for $20 \mathrm{~min}$ at room temperature. After two washes with PBS to remove excess stain the coverslips were mounted in VectaShield (Vector Laboratories, Burlingame, CA) and visualized using a Zeiss LSM 510 confocal microscope. TRITC and GFP were excited using a series of different lasers and the emissions for each collected, GFP was excited at $488 \mathrm{~nm}$ with an argon laser $(1 \%)$, and the emission was collected through a 505- to 550-nm barrier filter. TRITC was excited at $543 \mathrm{~nm}$ with a helium neon laser $(3 \%)$, and the emission was collected through $560-\mathrm{nm}$ long pass barrier filter. Images were collected and were processed using MetaMorph software (Molecular Devices, Sunnyvale, CA).

For immunocytochemistry, $72 \mathrm{~h}$ induced versus noninduced cells were fixed with $4 \%$ paraformaldehyde for $30 \mathrm{~min}$, permeabilized with $0.5 \%$ Triton
X-100 for $20 \mathrm{~min}$, and blocked for $1 \mathrm{~h}$ with $5 \%$ bovine serum albumin (BSA) We used anti-TAGLN antibody (1:50, $1 \mathrm{~h}$, Ab14106; Abcam, Cambridge, United Kingdom) and Cy3-conjugated anti-rabbit antibody $(1: 800,1 \mathrm{~h}, 111$ 166-045; Jackson ImmunoResearch Laboratories, West Grove, PA) to label Transgelin. Images were taken with a Zeiss confocal microscope (Carl Zeiss, Jena, Germany)

\section{Preparation and Transfection of Primary Cortical Neurons}

Primary cultures of cortical neurons were prepared from embryonic day 19-20 C57 BL6J mice. Cerebral cortices were collected and maintained in ice-cold $\mathrm{Ca}^{2+} / \mathrm{Mg}^{2+}$-free PBS followed by incubation in $0.25 \mathrm{mg} / \mathrm{ml}$ trypsin PBS supplemented with $1.5 \mathrm{mM}$ glucose. Trypsin was inactivated by inactivation with $0.25 \mathrm{mg} / \mathrm{ml}$ soybean trypsin inhibitor (SBTI). The supernatant was aspirated, and the cells were triturated in PBS containing SBTI by using a 1-ml pipette until all the clumps were broken up. Cells were pelleted at $200 \times g$ for $2 \mathrm{~min}$ and resuspended in minimum essential media (Invitrogen, Biosciences Ireland, Dublin, Ireland) containing 25\% horse serum, 25\% fetal calf serum, $0.5 \mathrm{mM}$ L-glutamine, $100 \mathrm{units} / \mathrm{ml}$ penicillin, and $100 \mu \mathrm{g} / \mathrm{ml}$ streptomycin. Neurons were plated on poly-D-lysine-coated glass coverslips at a concentration of 750,000 cells $/ \mathrm{ml}$ and kept at $37^{\circ} \mathrm{C}$ in a $5 \% \mathrm{CO}_{2}$ incubator. After $2-3 \mathrm{~h}$ in vitro, the initial plating medium was diluted with an equal volume of maintenance medium (Neurobasal E medium [Invitrogen] plus 0.4 $\mu \mathrm{g} / \mathrm{ml}$ glutamate and 2\% B27 supplement [Invitrogen]). Neurons were transfected with pIRES-AICD50-EGFP and pCI-Neo-Fe65 or pIRES-EGFP and pCI-Neo control vector constructs on day 2 in vitro by using the calcium phosphate method.

\section{RESULTS}

\section{Generation of Stable Cell Lines Inducible for AICD, FE65, and Both AICD and FE65}

To investigate potential AICD-dependent targets, we established an inducible AICD expression system (abbreviated $\mathrm{iA}$, inducible for AICD) in the human neuroblastoma cell line SHEP-SF. Similar to other neuroblastoma cells, SHEP-SF cells express endogenous FE65 and TIP60 (Figure 1B). Given that the expression levels of FE65 have been suggested to be an important threshold for potentiating transactivation by AICD (Cao and Sudhof, 2001), we also established stable cell lines inducible for FE65 (iF) or both FE65 and AICD (iFA) together. Coexpression of EGFP was used as selection marker in all stable cell lines, with inducible EGFP cell lines (iEGFP) established as controls. Processing of APP through $\gamma$-secretase activity results in the release of different AICD isoforms corresponding to cleavage at the $\gamma$-(AICD59) or $\varepsilon$ (AICD50)-site (Figure 1A). We therefore generated inducible stable cell lines expressing either isoform AICD59 or AICD50 (iA50, iA59, iFA50, and iFA59 stable cell lines).

Established cell lines were characterized for induction of ectopically inserted genes on the mRNA level (Figure 2, A and B). To differentiate between AICD50 and AICD59 isoforms, two different primer sets were used in competitive reverse transcription (RT)-PCR. Induction of AICD and FE65 was evident 72-h post-dox withdrawal (Figure 2A). Earlier time points after the dox washout did not reveal high induction levels of AICD, suggesting dox traces remaining in the cells still sufficient to repress induction of ectopically inserted genes. Indeed, significant AICD or FE65 induction was absent $48 \mathrm{~h}$ after dox removal (data not shown). Realtime quantitative PCR demonstrated high induction levels of AICD and/or FE65 with small interclonal variations (Figure $2 \mathrm{~B}$ ). Induction of AICD resulted in a 23-fold increase in both iA (23.0 \pm 4.8-fold) and iFA $(23.5 \pm 4.0)$ clones. A similar induction level was found for FE65 in iF (20.1 \pm 2.4 -fold) and iFA (16.3 \pm 8.1 -fold) clones. This potent induction of AICD and FE65 was also confirmed at the protein level by Western blotting (Figure 2C). Of note, AICD protein levels detected by conventional Western blotting could lead to an underestimation of actual AICD expression, because we did not use antigen retrieval (Ryan and Pimplikar, 2005). 

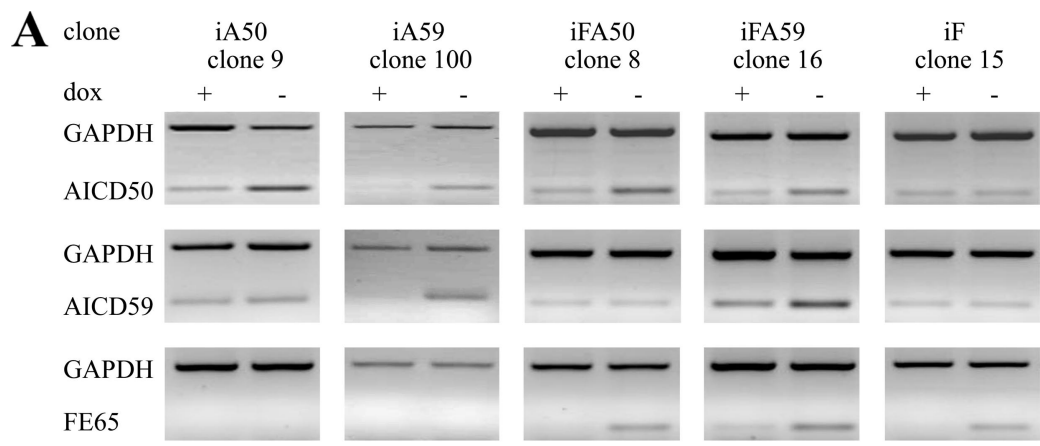

B
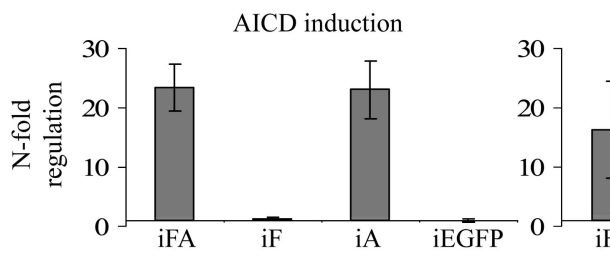

FE65 induction
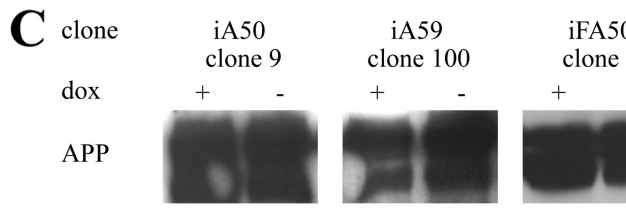

$$
\text { clone } 8
$$

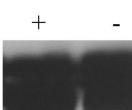

$-97.4 \mathrm{kDa}$
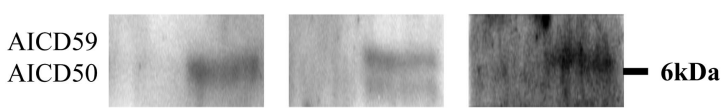

ß Actin

FE65

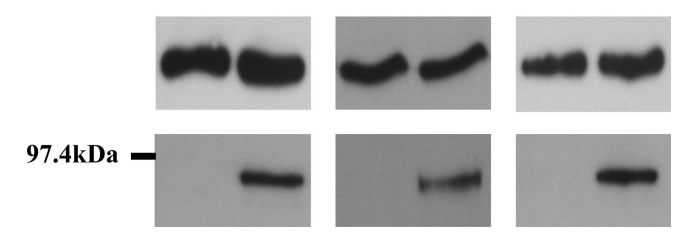

Figure 2. Characterization of inducible cell lines. (A) RNA was isolated from 72-h-induced (-dox) and noninduced (+dox; as control) cell lines iA50, iA59, iFA50, iFA59, and iF and analyzed for induction of ectopic-inserted genes by competitive RT-PCR. Two sets of primer pairs were used to differentiate between AICD50 and AICD59 isoforms. (B) Quantification of induction levels by quantitative real-time PCR revealed an average AICD induction in $\mathrm{iA}$ clones of $23.04 \pm 4.78$ and $23.47 \pm 3.95$ in iFA clones. FE65 induction was $16.3 \pm 8.1 \mathrm{in} \mathrm{iFA}$ and $20.11 \pm 2.36 \mathrm{in} \mathrm{iF}$ clones. We analyzed each two iA50, iA59, iFA50, and iFA59 clones, three iF clones, and three iEGFP clones (figure shows averaged induction in each case). (C) Western blot analysis of whole cell lysates verified specific induction of AICD and FE65, respectively. AICD was detectable after immunoprecipitation. APP signal was used as control.
Together, we were able to establish a human neuroblastoma cell culture model expressing the intracellular domain of APP and its binding protein FE65 in an inducible manner suitable to study the transcriptional potential of AICD.

\section{AICD-dependent Induction of Differential Gene Expression}

To identify AICD-dependent gene targets, we analyzed the transcriptional changes occurring after induction of AICD alone or AICD in combination with FE65 by using a microarray approach. Cells were induced for $72 \mathrm{~h}$, and cRNA from two iA50 clones and one iFA50 clone were compared versus the noninduced cells by using the Affymetrix GeneChips U133A made up of $>22,000$ transcripts. After data analysis with the GeneData Expressionist software, we identified 182 differentially regulated genes that showed regulation in iA clones, iFA clones, or both iA and iFA clones (see Supplemental Table). Genes were selected on the basis of highest expression ratio or putative relevance in $\mathrm{AD}$ and further analyzed using quantitative real-time PCR. Analysis was performed for the iA50 and iFA50 clones (which were used for the microarray analysis) as well as for one iA59, another iFA50, one iFA59, two iF, and two iEGFP clones. In total, identification of AICD-dependent target genes was based on the analysis of 10 different clones.

Eight selected genes were confirmed by quantitative realtime PCR as differentially regulated in an AICD-dependent manner (Table 1). There was little variety in the respective expression level within the individual three iFA, three iA, two iF, or two iEGFP clones (Table 1). The microarray data failed to identify differential expression of the previously described AICD targets, including genes KAI1, GSK3 $\beta$, SERCA2B, APP, and Neprilysin, which were present on the U133A GeneChip. Moreover, real-time quantitative PCR analysis confirmed that AICD or AICD/FE65 expression was not associated with changes in expression of either KAI1, GSK3 $\beta$, or SERCA2B (Table 2). Interestingly, expression levels of AICD-dependent target genes were very similar after overexpression of the AICD50 or AICD59 isoform (Figure 3).

\section{Different Regulation Pattern of AICD-dependent Target Genes}

Comparison of induction levels of AICD targets in the iA and iFA clones revealed three different regulation patterns. One group of AICD-dependent regulated genes (group I: insulin growth factor binding protein 3 [IGFBP3], solute carrier family 7, member 5 [SLC7A5]) showed similar levels of induction in both iA and in iFA clones, suggesting that FE65 induction does not influence expression of these genes. Other genes (group II: ACTA2, Fibronectin1 [FN1], Transgelin [TAGLN, SM22], Tropomyosin 1 [TPM1]) revealed changes in their expression level under AICD induction (iA 
Table 1. AICD-dependent target genes found in this work

\begin{tabular}{|c|c|c|c|c|c|c|c|c|c|c|}
\hline Gene & $\begin{array}{l}\text { iFA50 } \\
\text { clone } 6\end{array}$ & $\begin{array}{l}\text { iFA50 } \\
\text { clone } 8\end{array}$ & $\begin{array}{c}\text { iFA59 } \\
\text { clone } 16\end{array}$ & $\begin{array}{l}\text { iA50 } \\
\text { clone } 6\end{array}$ & $\begin{array}{l}\text { iA50 } \\
\text { clone } 9\end{array}$ & $\begin{array}{c}\text { iA59 } \\
\text { clone } 100\end{array}$ & $\begin{array}{c}\mathrm{iF} \\
\text { clone } 5\end{array}$ & $\begin{array}{c}\mathrm{iF} \\
\text { clone } 15\end{array}$ & $\begin{array}{l}\text { iEGFP } \\
\text { clone } 9\end{array}$ & $\begin{array}{c}\text { iEGFP } \\
\text { clone } 22\end{array}$ \\
\hline \multicolumn{11}{|c|}{ IGFBP3 } \\
\hline RF & 5.9 & 4.82 & 4.7 & 5.72 & 5.03 & 4.77 & 1.87 & 1.83 & 1.09 & 0.89 \\
\hline SD & 0.29 & 0.06 & 1.3 & 0.26 & 0.26 & 0.64 & 0.09 & 0.74 & 0.03 & 0.15 \\
\hline \multicolumn{11}{|c|}{ TAGLN } \\
\hline RF & 3.31 & 4.74 & 3.86 & 5.10 & 1.91 & 2.79 & 1.92 & 1.95 & 0.87 & 0.89 \\
\hline SD & 1.17 & 0.44 & 0.61 & 0.83 & 0.21 & 0.09 & 0.35 & 0.65 & 0.01 & 0.08 \\
\hline \multicolumn{11}{|c|}{ ACTA2 } \\
\hline RF & 2.60 & 3.61 & 2.02 & 2.38 & 1.78 & 1.37 & 1.06 & 0.93 & 0.89 & 1.08 \\
\hline SD & 0.86 & 0.33 & 0.48 & 0.19 & 0.31 & 0.21 & 0.15 & 0.40 & 0.21 & 0.15 \\
\hline \multicolumn{11}{|l|}{ FN1 } \\
\hline RF & 3.19 & 2.25 & 1.7 & 1.36 & 1.07 & 1.63 & 1.25 & 1.09 & 0.80 & 0.72 \\
\hline SD & 0.67 & 0.12 & 0.21 & 0.2 & 0.08 & 0.11 & 0.06 & 0.08 & 0.04 & 0.06 \\
\hline \multicolumn{11}{|c|}{ RAB3B } \\
\hline RF & 3.13 & 2.06 & 1.59 & 1.47 & 0.78 & 1.49 & 1.43 & 1.48 & 1.01 & 1.16 \\
\hline SD & 0.60 & 0.06 & 0.32 & 0.09 & 0.08 & 0.04 & 0.09 & 0.37 & 0.07 & 0.12 \\
\hline \multicolumn{11}{|l|}{ TPM1 } \\
\hline RF & 3.00 & 2.08 & 1.54 & 1.77 & 1.33 & 1.38 & 1.29 & 0.83 & 1.10 & 1.08 \\
\hline SD & 0.77 & 0.18 & 0.13 & 0.23 & 0.12 & 0.26 & 0.13 & 0.05 & 0.38 & 0.04 \\
\hline \multicolumn{11}{|c|}{ SLC7A5 } \\
\hline RF & 2.35 & 1.66 & 1.54 & 1.27 & 1.69 & 2.08 & 1.23 & 1.33 & 1.04 & 0.96 \\
\hline SD & 0.44 & 0.11 & 0.18 & 0.07 & 0.1 & 0.05 & 0.16 & 0.58 & 0.07 & 0.16 \\
\hline \multicolumn{11}{|c|}{ MICAL2 } \\
\hline RF & 2.24 & 1.53 & 1.45 & 0.76 & 0.67 & 0.93 & 1.08 & 1.08 & 1.18 & 0.97 \\
\hline SD & 0.51 & 0.11 & 0.23 & 0.08 & 0.06 & 0.11 & 0.09 & 0.40 & 0.10 & 0.24 \\
\hline
\end{tabular}

Genes were originally identified using Affymetrix GeneChips comparing induced (72 h) versus noninduced cells of two iA50 clones and one iFA50 clone. Selected genes were further analyzed using SYBR Green real-time PCR. Finally, we found eight genes that were AICDdependent regulated. The table shows regulation factors of three iFA clones ( 2 iFA50 and 1 iFA59 clones), three iA clones (2 iA50 and 1 iA59 clones), two iF clones, and two iEGFP clones. Experiments were performed on two independent dates in quadruplicate. RF, regulation factor; SD, SD for calculation, method see Materials and Methods).

clones), but these changes were enhanced upon cooverexpression of FE65. Finally, a third group of genes (group III: flavoprotein oxidoreductase MICAL2 [MICAL2], Ras-associated protein $[\mathrm{RAB} 3 \mathrm{~B}])$ only showed differential expression after induction of both AICD and FE65. Together, our approach revealed a transcriptional potential

Table 2. KAI1, GSK3 $\beta$, and SERCA2B regulation

\begin{tabular}{lcccc}
\hline & \multicolumn{5}{c}{-Fold regulation factor } \\
\cline { 2 - 5 } Gene & $\mathrm{iFA}$ & $\mathrm{iF}$ & $\mathrm{iA}$ & $\mathrm{iEGFP}$ \\
\hline KAI1 & & & & \\
$\quad$ RF & 0.94 & 0.80 & 0.57 & 0.74 \\
SD & 0.02 & 0.09 & 0.15 & 0.03 \\
GSK3 $\beta$ & & & & \\
$\quad$ RF & 0.91 & 1.06 & 1.14 & 1.19 \\
$\quad$ SD & 0.14 & 0.26 & 0.06 & 0.23 \\
SERCA2B, ATPase, Ca ${ }^{++}$ & & & & \\
transporting, slow-twitch & & & & \\
$\quad$ RF & 1.08 & 1.00 & 0.94 & 0.92 \\
$\quad$ SD & 0.16 & 0.16 & 0.10 & 0.32 \\
\hline
\end{tabular}

Expression of KAI1, GSK3 $\beta$, and SERCA2B in induced (-dox, $72 \mathrm{~h}$ ) versus noninduced (+dox) iFA, iF, iA, and iEGFP cells was analyzed using real-time PCR. Table shows average regulation factors of four iFA clones (2 iFA50 and 2 iFA59 clones), four iA clones (2 iA50 and 2 iA59 clones), three iF clones, and three iEGFP clones. Our analysis revealed no differential regulation for KAI1, GSK3 $\beta$, and SERCA2B. for AICD and mechanistically different gene expression changes depending on the presence of AICD and its binding protein FE65.

Control clones that were inducible for FE65 only or EGFP did not show any differential regulation for the described AICD target genes except for TAGLN and IGFBP3, which were slightly up-regulated in iF clones. Interestingly, TAGLN and IGFBP3 were the most strongly induced genes investigated, suggesting that endogenous AICD levels were sufficient to trigger the induction of both genes in FE65-induced cells.

\section{AICD-dependent Regulated Genes Are Involved in Cytoskeleton Dynamics}

A group of genes associated with the highest levels of induction were genes involved in the regulation of the cytoskeleton. ACTA2 (group II), TAGLN (group II), and TPM1 (group II) showed an increased expression in iA or iFA clones. These genes have central functions in the regulation of the actin cytoskeleton. Transgelin plays a role in the organization and stability of the actin cytoskeleton (Goodman et al., 2003). Tropomyosin 1 was originally described as protein interacting with actin filaments of myofibrils. Interestingly, it was recognized in neurofibrillary tangles, the intraneuronal hallmark of AD (Galloway et al., 1990). Other genes with potential roles in the regulation of the cytoskeleton and induced by AICD/FE65 included FN1 (group II), ras-associated protein $3 \mathrm{~B}$ (RAB3B, group III), and MICAL2 (group III) (Terman et al., 2002). 

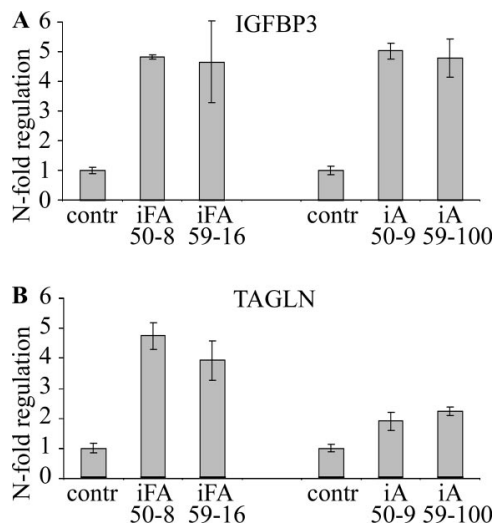

Figure 3. AICD target gene expression levels are elevated to a similar extent by both AICD isoforms. Quantitative real-time PCR analysis revealed upregulation of IGFBP3 (A) and TAGLN (B) target genes in iA (iA50 clone 9, iA59 clone 100) and iFA (iFA50 clone 8, iFA59 clone 16) clones. However, no differences were found between clones expressing AICD50 ( \pm FE65) or AICD59 ( \pm FE65) isoform. Relevance of AICD isoform was also not detectable for other target genes (Table 1) described in this work.

\section{Differential Expression of AICD-dependent Target Genes in Alzheimer's Disease Brains and in AICD/FE65-transfected Cortical Neurons}

We next investigated whether the identified AICD target genes were differentially regulated in the frontal gray matter, a brain region with neuropathological AD hallmarks, of AD patients $(n=5)$ versus age-matched controls $(n=6)$. Nondegraded RNA as determined by gel electrophoresis was used as template. Expression of both APP and FE65 mRNA was unaltered in control versus AD samples (Figure 4A). Interestingly, the three most prominently induced AICD target genes, $\alpha 2$-Actin, IGFBP3, and TAGLN, were significantly up-regulated in the frontal cortex of AD patients compared with age matched controls $(\alpha 2$-Actin, $\mathrm{p}=$ 0.001; IGFBP3, $p=0.005$; and TAGLN, $p=0.002$ ). In contrast, KAI1, GSK3 $\beta$, and SERCA2B were not differentially regulated (Figure 4A).

Given that APP expression is predominantly neuronal, AICD-dependent targets should therefore demonstrate neuronal expression. Immunohistochemical analysis of the lo- calization of the AICD target gene $\alpha 2$-Actin in hippocampal and frontal human brain slices revealed that $\alpha 2$-Actin immunopositivity was exclusively observed in neurons, and as expected in vessel walls, whereas glial cells did not show any staining (Figure 4B). Unfortunately poor antibody quality for paraffin-embedded tissue prevented any further immunohistochemical analyses of AICD-dependent genes.

To confirm several of the newly identified AICD targets in an independent cell system, we transiently transfected primary cortical neurons with AICD50 and FE65 expression vectors and harvested total RNA 48-h posttransfection. As a control, cells were mock transfected with the appropriate empty vectors. Despite low transfection efficiency (5-10\%; data not shown) real-time qPCR analysis demonstrated that AICD50/FE65 overexpression was associated with increased expression of TAGLN, TPM1, and ACTA2 compared with mock-transfected controls (Figure 4C).

\section{Overexpression of AICD and FE65 Increases Actin Dynamics}

The microarray data identified several genes involved in the regulation of actin dynamics. Therefore, we sought to investigate whether changes in the expression levels of AICD and FE65 reflected changes in the actin dynamics within the neuroblastoma cells. For this purpose, cells were induced to express AICD and FE65 for $72 \mathrm{~h}$. Using fluorescently labeled phalloidin, a high-affinity F-actin binding molecule, we examined the organization of actin filaments within the cell. In the noninduced iFA cells, the F-actin was primarily present in the form of parallel fibers (Figure 5A). In contrast to this organized filamentous structure, induction of AICD/FE65 (-dox) was associated with a destabilization of actin fibers within the body of the cell and clumping of actin at the periphery (Figure 5A). Importantly, this effect was not observed following induction of EGFP (Figure 5B) or FE65 alone (Figure 5C). Transgelin plays a role in the organization and stability of the actin cytoskeleton and was found differential regulated in induced versus noninduced iFA cells. Western blotting approved this regulation for Transgelin protein, which was 1.8 -fold $( \pm 0.07)$ increased in induced iFA cells versus noninduced cells (Figure 5E). Immunocytochemistry of Transgelin clearly revealed increased expression in induced cells versus noninduced cells (Figure 5F).

Transient transfection experiments in primary cultures of cortical neurons revealed similar results (Figure 5D). Whereas
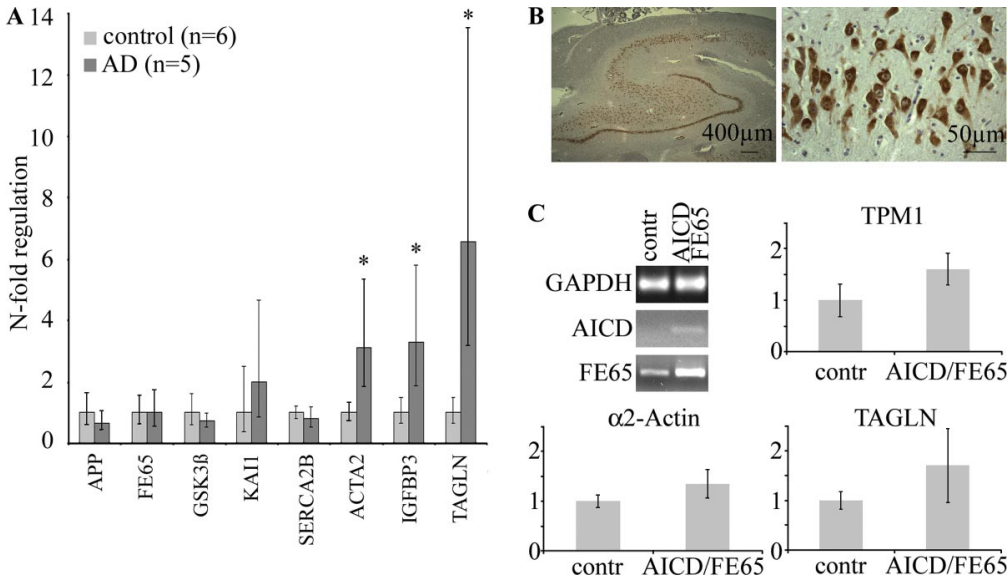

Figure 4. Analysis of AICD-dependent target genes in tissue of human brain samples and murine cortical neurons. (A) Isolated RNA from the frontal cortex of human $\mathrm{AD}$ and control brain tissue was analyzed by gel electrophoresis to calculate RNA degradation. Samples with no or low degradation were used for real-time PCR analysis resulting in five samples from $A D$ patients and six samples from control patients. $\alpha 2$-Actin $\left({ }^{*} \mathrm{p}=0.001\right)$ IGFBP3 $\left({ }^{*} p=0.005\right)$ and TAGLN $\left({ }^{*} p=0.002\right)$, which were the three most prominent AICD target genes found in our cell culture model, were significantly upregulated in AD brains versus controls. Statistical significance was calculated by using one-way ANOVA. KAI1 was not significantly differential regulated in AD brains versus controls. (B) Immunohistochemical stainings of human brain sections clearly revealed cytoplasmatic neuronal staining for ACTA2, here shown in the hippocampus. (C) Primary cortical neurons were transfected with AICD/FE65 expression vectors or control

empty vectors. Forty-eight hours posttransfection, cells were harvested and total RNA was extracted. Quantitative real-time PCR analysis showed increased expression of TAGLN, TPM1, and ACTA2 compared with controls. 
Figure 5. Overexpression of AICD/FE65 increases actin dynamics. iFA (A) or control iEGFP (B) cells were induced for $72 \mathrm{~h}$. Noninduced ( + dox) and induced ( - dox) cells were stained with rhodamine-phalloidin (red) to visualize F-actin. EGFP expression and rhodamine-labeled actin filaments were visualized by confocal microscopy. Induction of both AICD and FE65 revealed disorganization of actin fibers within the cell body and clumping of actin fibers at the periphery, suggesting increased actin dynamics (A). In contrast, induction of EGFP (B) of Fe65 (C) did not influence actin fibers. Bar, $20 \mu \mathrm{m}$. Images are representative for three independent experiments. (D) Overexpression of AICD/FE65 increases actin dynamics in cortical neurons. Primary cultures of cortical neurons were prepared from embryonic day 19-20 C57 BL6J mice and transfected on day 2 in vitro with AICD/FE65 or EGFP control vector. Neurons were stained and visualized in analogy to the neuroblastoma cells. Similar to our results in neuroblastoma cells, all cells expressing both AICD and FE65 revealed disorganization of actin fibers within the cell body and clumping of actin fibers at the periphery (arrowhead). In contrast, just $12 \%$ of EGFP control vector transfected cells showed the same phenotype, whereas the majority did not reveal cytoskeletal changes. Bar, $10 \mu \mathrm{m}$. (E) Western blotting of induced versus noninduced iFA clones revealed increased expression of Transgelin (TAGLN) protein in induced iFA cells, whereas control clones (iEGFP) showed similar expression. Quantification based on two different Western blots and revealed 1.8-fold $( \pm 0.07)$ up-regulation of TAGLN in induced versus noninduced iFA cells. (F) Up-regulation of TAGLN was also evident in induced versus noninduced iFA cells as illustrated by immunocytochemistry. Bar, $20 \mu \mathrm{m}$.

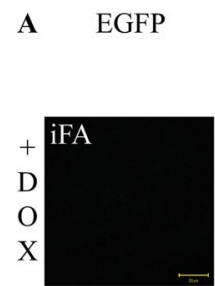

Rhodamine
phalloidin

merge

staining
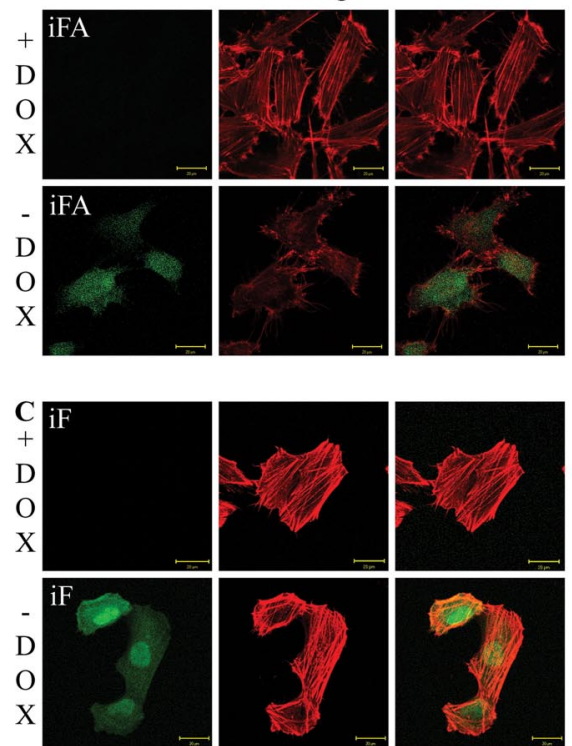

D EGFP
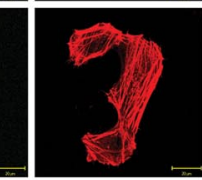

Rhodamine phalloidin staining
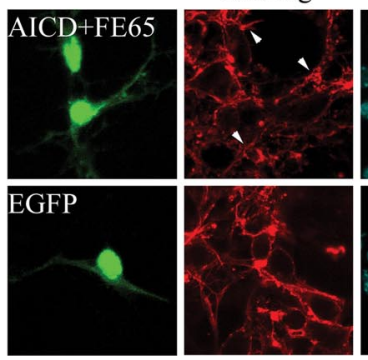

DAPI

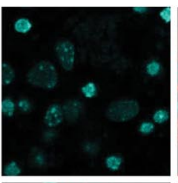

B EGFP
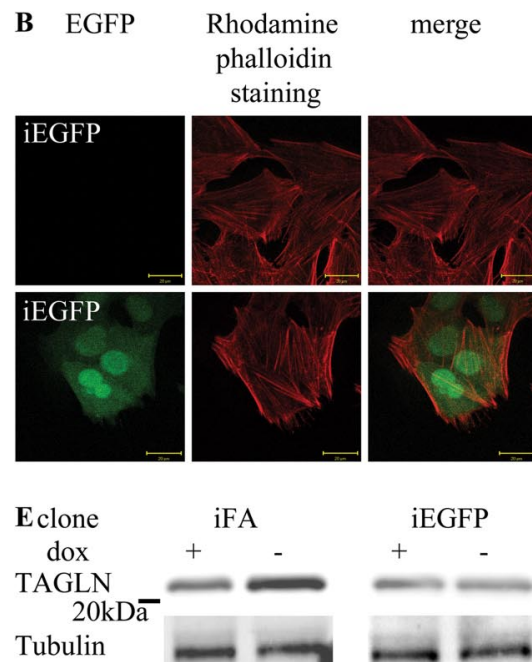

Tubulin

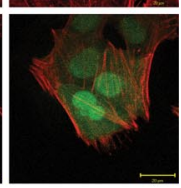

iEGFP $+$
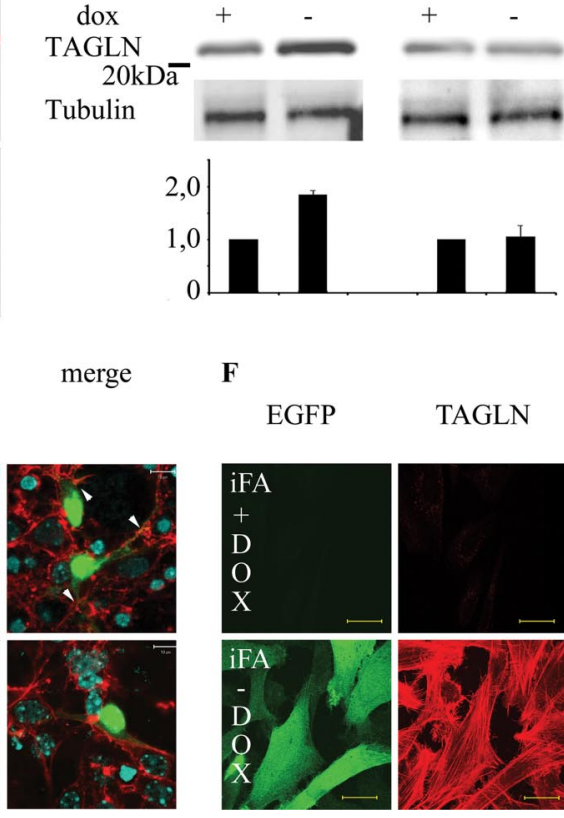

EGFP

TAGLN
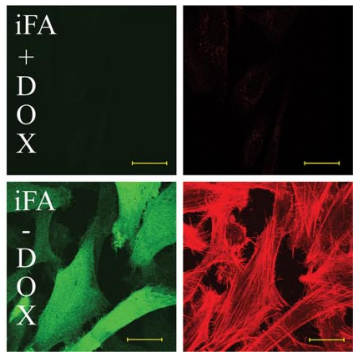

all AICD/FE65 transfected neurons showed clumping of actin at the periphery (Figure 5D, arrowhead), this effect was just observable in $12 \%$ of EGFP control vector-transfected cells. Together, these results suggest that AICD increases actin dynamics and may be involved in the reorganization of the cytoskeleton.

\section{DISCUSSION}

It has been suggested that AICD has an important biological role in the development of AD pathology through its ability to act as a cleavage product with transactivation potential (Cao and Sudhof, 2001). Until now, few AICDdependent candidate genes had been described, and a genome-wide approach to screening was lacking. In this study, we established an inducible neuroblastoma cell culture model with AICD, FE65, or AICD and FE65 under control of a tetracycline-responsive promoter. Analysis of transcriptional changes after expression of AICD and/or its cofactor FE65 by using Affymetrix U133A GeneChips revealed a list of 182 putative AICD target genes (see Supplemental Table).

Real-time quantitative PCR of selected genes demonstrated that some genes were dependent on induction of AICD alone; others showed elevated expression when FE65 was coexpressed, whereas a third subset was only differentially regulated after expression of both AICD and FE65.

Fe65-independent signaling might be explained by direct binding of AICD to TIP60 (Kinoshita et al., 2002). However, the majority of target genes described in this work demonstrated a requirement for coexpression of FE65, suggesting an important role for this binding protein in AICD-dependent gene expression, an observation that has also been reported by other studies (Cao and Sudhof, 2001; von Rotz et al., 2004). Indeed, there is evidence that binding of FE65 to AICD is necessary for the nuclear translocation (Kimberly et al., 2001; Muresan and Muresan, 2004; von Rotz et al., 2004). Two different AICD transcriptionally active complexes based on the binding of AICD-FE65 to TIP60 and to CP2/ LSF/LBP1 have been suggested (Cao and Sudhof, 2001; Kim et al., 2003). TIP60 is part of a large nuclear complex with DNA binding, ATPase and DNA helicase activity (Ikura et al., 2000). In Gal4 reporter gene experiments, the ternary complex AICD-FE65-TIP60 is a potent transactivator, dependent on the expression level of FE65 (Cao and Sudhof, 2001). An alternative mechanism based on structural modifications of FE65 by AICD has been suggested that does not require AICD translocation from the cytoplasm to nucleus (Cao and Sudhof, 2004). This hypothesis is not in contrast to our findings, because elevated cytosolic levels of AICD might be 
sufficient to cause a change in the conformation of FE65. This second ternary complex (AICD-FE65-CP2/LSF/LBP1) was suggested to modulate expression of glycogen synthase kinase-3 $\beta$ (Kim et al., 2003). Therefore, different AICD transcriptional complexes might explain different regulation patterns.

Long-time induction of ectopically expressed genes may result in secondary regulation effects as primary induced genes itself could act as activator for other genes. Therefore, we used 72-h induction (dox washout) for our studies corresponding to the earliest time point of measurable induction of AICD and/or FE65. Under these conditions we did not observe differential regulation of previously described AICD target genes such as KAI1 (Table 2). We could not detect regulation of other putative target genes, including SERCA2B (Leissring et al., 2002) or GSK3 $\beta$ (Kim et al., 2003). However, our data are in agreement with other groups who could not detect AICD-dependent regulation of these genes in AICD/FE65 transgenic mice (Ryan and Pimplikar, 2005) or in cell lines with pharmacological inhibition or deficiency of $\gamma$-secretase activity (Hebert et al., 2006). Furthermore, it is probable that differential gene expression of AICD targets occurs in a cell type-specific manner and is dependent on expression levels of endogenous cofactors. Our work is the first study analyzing AICD-dependent gene expression in stable transfected SHEP-SF neuroblastoma cells. Importantly, primary cortical neurons transiently transfected with AICD50/FE65 also demonstrated increased expression of TAGLN, TPM1 supporting the hypothesis of a role for AICD in regulating the expression levels of these genes. Moreover, given the emerging evidence for a role for AICD signaling in the pathophysiology of AD, we were able to verify differential expression of several of the AICD target genes described in this work in the frontal cortex of Alzheimer disease versus control brains. $\alpha 2$-Actin, IGFBP3, and TAGLN were significantly higher expressed in AD brains compared with their age-matched control. Interestingly, IGFBP3 has previously been described to be associated with neurofibrillary tangles in AD brains (Rensink et al., 2002). Furthermore, other groups have also reported differential expression of PRKC (Par-4) (El Guendy and Rangnekar, 2003) and TPM1 (Galloway et al., 1990) in AD brain samples compared with brains from unaffected patients. Moreover, neuritic plaques positive for neuregulin (Chaudhury et al., 2003) and fibronectin 1 (Van Gool et al., 1994) have been shown by immunohistochemistry. Given that several of our AICD target genes have previously demonstrated differential expression levels during AD progression, it is interesting to postulate that increased expression of AICD, and of its targets, might play an important role in AD. In contrast, we could not confirm differential regulation for KAI1 and other discussed target genes in AD brains versus controls.

Among the AICD-dependent regulated genes, we identified several candidates that have previously been described as regulators of actin dynamics, including $\alpha 2$-Actin, Transgelin, and Tropomyosin 1. Transgelin, an actin cross-linking protein, is involved in the organization of the actin cytoskeleton and is thought to link aging to actin stability (Goodman et al., 2003; Nakano et al., 2005). Other genes as Fibronectin1 and RAB3B were also described to be associated with cytoskeletal organization. Visualization of F-actin structures in neuroblastoma cells as well as in cortical neurons after AICD and FE65 expression revealed disorganization of actin fibers within the cell body and clumping of actin fibers at the periphery, suggesting increased actin dynamics. Given that this phenotype was absent in cells expressing FE65 without AICD, our results suggest that this signaling occurs inde- pendent of the family of Ena/Vasp proteins, which interact with the WW domains of FE65 proteins (Ermekova et al., 1997). In contrast, our data suggest that AICD dependent transactivation is responsible for the reorganization of actin filaments, and hence may play an important role during development and in reorganization of cellular structures in response to injury. Moreover, APP itself is transcriptionally up-regulated in response to nerve transections, trauma, and cerebral ischemia in the nervous system (Banati et al., 1993; Shi et al., 2000; Ciallella et al., 2002), all processes that result in cytoskeletal reorganization. In Drosophila, APP expression is associated with increases in postdevelopmental axonal arborization and loss of neuronal connections, which was strictly linked to the intracellular domain of APP (Leyssen et al., 2005). Moreover, Tg2576 transgenic mice from as early as 4 mo old are positive for rod-like inclusions in the posterior cortex which are composed of actin (Maloney et al., 2005). A model in which APP and FE65 are involved in the complex regulation of actin-based growth cone motility is also supported by other data (Sabo et al., 2003). Interestingly, blockage of axonal transport, which might be a result of disorganized cytoskeleton, is the earliest measured disturbance in the brains of transgenic mice expressing mutant human APP (Stokin et al., 2005). Furthermore, recent studies demonstrate that meningeal fibroblasts from $\mathrm{FE}^{-/-}$plus F65L1-/mice reveal a disturbed F-actin pattern compared with wildtype mice (Guenette et al., 2006). Together, our study suggests an important role of AICD in the regulation of cellular actin dynamics.

\section{ACKNOWLEDGMENTS}

We thank C. Kaether, T. Russo, M. Schwab, and D. J. Selkoe for constructs, antibodies, and cell lines as well as Susanne Peetz-Dienhart for technical assistance. This study was supported by Innovative Medizinische Forschung Grant EG 120301 (to R.E.), the Health Research Board Grant RP/2004/125 (to J.H.M.P.), and Alzheimer Forschung Initiative e.V. 03809 (to D.K. and R.E.).

\section{REFERENCES}

Artavanis-Tsakonas, S., Rand, M. D., and Lake, R. J. (1999). Notch signaling: cell fate control and signal integration in development. Science 284, 770-776.

Baek, S. H., Ohgi, K. A., Rose, D. W., Koo, E. H., Glass, C. K., and Rosenfeld, M. G. (2002). Exchange of N-CoR corepressor and Tip60 coactivator complexes links gene expression by NF-kappa B and beta-amyloid precursor protein. Cell 110, 55-67.

Banati, R. B., Gehrmann, J., Czech, C., Monning, U., Jones, L. L., Konig, G., Beyreuther, K., and Kreutzberg, G. W. (1993). Early and rapid de novo synthesis of Alzheimer beta A4-amyloid precursor protein (APP) in activated microglia. Glia 9, 199-210.

Bertrand, E., Brouillet, E., Caille, I., Bouillot, C., Cole, G. M., Prochiantz, A., and Allinquant, B. (2001). A short cytoplasmic domain of the amyloid precursor protein induces apoptosis in vitro and in vivo. Mol. Cell Neurosci. 18, 503-511.

Braak, H., and Braak, E. (1991). Neuropathological staging of Alzheimerrelated changes. Acta Neuropathol. 82, 239-259.

Cao, X., and Sudhof, T. C. (2001). A transcriptionally [correction of transcriptively] active complex of APP with Fe65 and histone acetyltransferase Tip60. Science 293, 115-120.

Cao, X., and Sudhof, T. C. (2004). Dissection of amyloid-beta precursor protein-dependent transcriptional transactivation. J. Biol. Chem. 279, 2460124611.

Chaudhury, A. R., Gerecke, K. M., Wyss, J. M., Morgan, D. G., Gordon, M. N., and Carroll, S. L. (2003). Neuregulin-1 and erbB4 immunoreactivity is associated with neuritic plaques in Alzheimer disease brain and in a transgenic model of Alzheimer disease. J. Neuropathol. Exp. Neurol. 62, 42-54.

Ciallella, J. R., Ikonomovic, M. D., Paljug, W. R., Wilbur, Y. I., Dixon, C. E. Kochanek, P. M., Marion, D. W., and DeKosky, S. T. (2002). Changes in expression of amyloid precursor protein and interleukin-1beta after experimental traumatic brain injury in rats. J. Neurotrauma 19, 1555-1567. 
Cupers, P., Orlans, I., Craessaerts, K., Annaert, W., and De Strooper, B. (2001). The amyloid precursor protein (APP)-cytoplasmic fragment generated by gamma-secretase is rapidly degraded but distributes partially in a nuclear fraction of neurones in culture. J. Neurochem. 78, 1168-1178.

Edbauer, D., Willem, M., Lammich, S., and Steiner, H. (2002). Insulin-degrading enzyme rapidly removes the beta-amyloid precursor protein intracellular domain. Neurobiol. Aging 23, S559

El Guendy, N., and Rangnekar, V. M. (2003). Apoptosis by Par-4 in cancer and neurodegenerative diseases. Exp. Cell Res. 283, 51-66.

Ermekova, K. S., Zambrano, N., Linn, H., Minopoli, G., Gertler, F., Russo, T., and Sudol, M. (1997). The WW domain of neural protein FE65 interacts with proline-rich motifs in Mena, the mammalian homolog of Drosophila enabled. J. Biol. Chem. 272, 32869-32877.

Farris, W., Mansourian, S., Chang, Y., Lindsley, L., Eckman, E. A., Frosch, M. P., Eckman, C. B., Tanzi, R. E., Selkoe, D. J., and Guenette, S. (2003). Insulin-degrading enzyme regulates the levels of insulin, amyloid beta-protein, and the beta-amyloid precursor protein intracellular domain in vivo. Proc. Natl. Acad. Sci. 100, 4162-4167.

Galloway, P. G., Mulvihill, P., Siedlak, S., Mijares, M., Kawai, M., Padget, H., Kim, R., and Perry, G. (1990). Immunochemical demonstration of tropomyosin in the neurofibrillary pathology of Alzheimer's disease. Am. J. Pathol. 137, 291-300.

Gao, Y., and Pimplikar, S. W. (2001). The gamma-secretase-cleaved C-terminal fragment of amyloid precursor protein mediates signaling to the nucleus. Proc. Natl. Acad. Sci. 98, 14979-14984.

Goodman, A., Goode, B. L., Matsudaira, P., and Fink, G. R. (2003). The Saccharomyces cerevisiae calponin/transgelin homolog Scp1 functions with fimbrin to regulate stability and organization of the actin cytoskeleton. Mol. Biol. Cell 14, 2617-2629.

Guenette, S., Chang, Y., Hiesberger, T., Richardson, J. A., Eckman, C. B., Eckman, E. A., Hammer, R. E., and Herz, J. (2006). Essential roles for the FE65 amyloid precursor protein-interacting proteins in brain development. EMBO J. $25,420-431$

Hartley, D. M., Walsh, D. M., Ye, C. P., Diehl, T., Vasquez, S., Vassilev, P. M., Teplow, D. B., and Selkoe, D. J. (1999). Protofibrillar intermediates of amyloid beta-protein induce acute electrophysiological changes and progressive neurotoxicity in cortical neurons. J. Neurosci. 19, 8876-8884.

Hebert, S. S., Serneels, L., Tolia, A., Craessaerts, K., Derks, C., Filippov, M. A., Muller, U., and Strooper, B. D. (2006). Regulated intramembrane proteolysis of amyloid precursor protein and regulation of expression of putative target genes. ЕMBO Rep. 7, 739-745.

Ikura, T., Ogryzko, V. V., Grigoriev, M., Groisman, R., Wang, J., Horikoshi, M., Scully, R., Qin, J., and Nakatani, Y. (2000). Involvement of the TIP60 histone acetylase complex in DNA repair and apoptosis. Cell 102, 463-473.

Kim, H. S., et al. (2003). C-terminal fragments of amyloid precursor protein exert neurotoxicity by inducing glycogen synthase kinase-3beta expression. FASEB J. 17, 1951-1953.

Kimberly, W. T., Zheng, J. B., Guenette, S. Y., and Selkoe, D. J. (2001). The intracellular domain of the beta-amyloid precursor protein is stabilized by Fe65 and translocates to the nucleus in a notch-like manner. J. Biol. Chem. 276, $40288-40292$.

Kinoshita, A., Whelan, C. M., Berezovska, O., and Hyman, B. T. (2002). The gamma secretase-generated carboxyl-terminal domain of the amyloid precursor protein induces apoptosis via Tip60 in H4 cells. J. Biol. Chem. 277, $28530-28536$.

Leissring, M. A., et al. (2002). A physiologic signaling role for the gammasecretase-derived intracellular fragment of APP. Proc. Natl. Acad. Sci. USA 99, 4697-4702

Leyssen, M., Ayaz, D., Hebert, S. S., Reeve, S., De Strooper, B., and Hassan, B. A. (2005). Amyloid precursor protein promotes post-developmental neurite arborization in the Drosophila brain. EMBO J. 24, 2944-2955.

Livak, K. J., and Schmittgen, T. D. (2001). Analysis of relative gene expression data using real-time quantitative PCR and the 2(-Delta Delta C(T)) Method. Methods 25, 402-408.

Maloney, M. T., Minamide, L. S., Kinley, A. W., Boyle, J. A., and Bamburg, J. R. (2005). beta-Secretase-cleaved amyloid precursor protein accumulates at actin inclusions induced in neurons by stress or amyloid beta: a feedforward mechanism for Alzheimer's disease. J. Neurosci. 25, 11313-11321.

Mattson, M. P. (2004). Pathways towards and away from Alzheimer's disease. Nature 430, 631-639.

McLoughlin, D. M., and Miller, C. C. (1996). The intracellular cytoplasmic domain of the Alzheimer's disease amyloid precursor protein interacts with phosphotyrosine-binding domain proteins in the yeast two-hybrid system. FEBS Lett. 397, 197-200.

Mirra, S. S., Heyman, A., McKeel, D., Sumi, S. M., Crain, B. J., Brownlee, L. M Vogel, F. S., Hughes, J. P., van Belle, G., and Berg, L. (1991). The consortium to establish a registry for Alzheimer's disease (CERAD). Part II. Standardization of the neuropathologic assessment of Alzheimer's disease. Neurology 41, 479-486.

Munoz, D. G., Wang, D., and Greenberg, B. D. (1993). Hirano bodies accumulate C-terminal sequences of beta-amyloid precursor protein (beta-APP) epitopes. J. Neuropathol. Exp. Neurol. 52, 14-21.

Muresan, Z., and Muresan, V. (2004). A phosphorylated, carboxy-terminal fragment of beta-amyloid precursor protein localizes to the splicing factor compartment. Hum. Mol. Genet. 13, 475-488.

Nakano, K., Bunai, F., and Numata, O. (2005). Stg 1 is a novel SM22/ transgelin-like actin-modulating protein in fission yeast. FEBS Lett. 579, 63116316.

Nunan, J., Shearman, M. S., Checler, F., Cappai, R., Evin, G., Beyreuther, K., Masters, C. L., and Small, D. H. (2001). The C-terminal fragment of the Alzheimer's disease amyloid protein precursor is degraded by a proteasomedependent mechanism distinct from gamma-secretase. Eur. J. Biochem. 268, 5329-5336.

Nunan, J., Williamson, N. A., Hill, A. F., Sernee, M. F., Masters, C. L., and Small, D. H. (2003). Proteasome-mediated degradation of the C-terminus of the Alzheimer's disease beta-amyloid protein precursor: effect of C-terminal truncation on production of beta-amyloid protein. J. Neurosci. Res. 74, $378-$ 385.

Octave, J. N., Essalmani, R., Tasiaux, B., Menager, J., Czech, C., and Mercken, L. (2000). The role of presenilin- 1 in the gamma-secretase cleavage of the amyloid precursor protein of Alzheimer's disease. J. Biol. Chem. 275, 15251528.

Pardossi-Piquard, R., et al. (2005). Presenilin-dependent transcriptional control of the Abeta-degrading enzyme neprilysin by intracellular domains of betaAPP and APLP. Neuron 46, 541-554.

Rensink, A. A., Gellekink, H., Otte-Holler, I., ten Donkelaar, H. J., de Waal, R. M., Verbeek, M. M., and Kremer, B. (2002). Expression of the cytokine leukemia inhibitory factor and pro-apoptotic insulin-like growth factor binding protein-3 in Alzheimer's disease. Acta Neuropathol. 104, 525-533.

Ryan, K. A., and Pimplikar, S. W. (2005). Activation of GSK-3 and phosphorylation of CRMP2 in transgenic mice expressing APP intracellular domain. J. Cell Biol. 171, 327-335.

Sabo, S. L., Ikin, A. F., Buxbaum, J. D., and Greengard, P. (2003). The amyloid precursor protein and its regulatory protein, FE65, in growth cones and synapses in vitro and in vivo. J. Neurosci. 23, 5407-5415.

Sastre, M., Steiner, H., Fuchs, K., Capell, A., Multhaup, G., Condron, M. M., Teplow, D. B., and Haass, C. (2001). Presenilin-dependent gamma-secretase processing of beta-amyloid precursor protein at a site corresponding to the S3 cleavage of Notch. EMBO Rep. 2, 835-841.

Selkoe, D. J. (1994). Normal and abnormal biology of the beta-amyloid precursor protein. Annu. Rev. Neurosci. 17, 489-517.

Shi, J., Yang, S. H., Stubley, L., Day, A. L., and Simpkins, J. W. (2000) Hypoperfusion induces overexpression of beta-amyloid precursor protein mRNA in a focal ischemic rodent model. Brain Res. 853, 1-4.

Stokin, G. B., Lillo, C., Falzone, T. L., Brusch, R. G., Rockenstein, E., Mount, S. L., Raman, R., Davies, P., Masliah, E., Williams, D. S., and Goldstein, L. S. (2005). Axonopathy and transport deficits early in the pathogenesis of Alzheimer's disease. Science 307, 1282-1288.

Terman, J. R., Mao, T., Pasterkamp, R. J., Yu, H. H., and Kolodkin, A. L. (2002). MICALs, a family of conserved flavoprotein oxidoreductases, function in plexin-mediated axonal repulsion. Cell 109, 887-900.

Van Gool, D., Carmeliet, G., Triau, E., Cassiman, J. J., and Dom, R. (1994). Appearance of localized immunoreactivity for the alpha 4 integrin subunit and for fibronectin in brains from Alzheimer's, Lewy body dementia patients and aged controls. Neurosci. Lett. 170, 71-73.

von Rotz, R. C., Kohli, B. M., Bosset, J., Meier, M., Suzuki, T., Nitsch, R. M., and Konietzko, U. (2004). The APP intracellular domain forms nuclear multiprotein complexes and regulates the transcription of its own precursor. J. Cell Sci. 117, 4435-4448.

Walsh, D. M., Fadeeva, J. V., Lavoie, M. J., Paliga, K., Eggert, S., Kimberly, W. T., Wasco, W., and Selkoe, D. J. (2003). gamma-Secretase cleavage and binding to FE65 regulate the nuclear translocation of the intracellular $\mathrm{C}$ terminal domain (ICD) of the APP family of proteins. Biochemistry 42, 66646673. 
T. Müller et al.

Walsh, D. M., Klyubin, I., Shankar, G. M., Townsend, M., Fadeeva, J. V., Betts, V., Podlisny, M. B., Cleary, J. P., Ashe, K. H., Rowan, M. J., and Selkoe, D. J. (2005). The role of cell-derived oligomers of Abeta in Alzheimer's disease and avenues for therapeutic intervention. Biochem. Soc. Trans. 33, 1087-1090.

Yu, C., Kim, S. H., Ikeuchi, T., Xu, H., Gasparini, L., Wang, R., and Sisodia, S. S. (2001). Characterization of a presenilin-mediated amyloid precursor protein carboxyl-terminal fragment gamma. Evidence for distinct mechanisms involved in gamma-secretase processing of the APP and Notch1 transmembrane domains. J. Biol. Chem. 276, 43756-43760.

Zambrano, N., Minopoli, G., de Candia, P., and Russo, T. (1998). The Fe65 adaptor protein interacts through its PID1 domain with the transcription factor CP2/LSF/LBP1. J. Biol. Chem. 273, 20128-20133. 